\title{
Nonstandard Analysis and Jump Conditions for Converging Shock Waves
}

\author{
by \\ Roy S. Baty \\ Los Alamos National Laboratory, Los Alamos, New Mexico 87545 \\ F. Farassat \\ NASA Langley Research Center, Hampton, Virginia 23681-2199 \\ Don H. Tucker \\ University of Utah, Salt Lake City, Utah 84112-0090
}

\begin{abstract}
Nonstandard analysis is an area of modern mathematics which studies abstract number systems containing both infinitesimal and infinite numbers. This article applies nonstandard analysis to derive jump conditions for one-dimensional, converging shock waves in a compressible, inviscid, perfect gas. It is assumed that the shock thickness occurs on an infinitesimal interval and the jump functions in the thermodynamic and fluid dynamic parameters occur smoothly across
\end{abstract}


this interval. Predistributions of the Heaviside function and the Dirac delta measure are introduced to model the flow parameters across a shock wave. The equations of motion expressed in nonconservative form are then applied to derive unambiguous relationships between the jump functions for the flow parameters.

\section{Introduction}

Currently, no known mathematical theory provides a general framework to study all the partial differential equations of mathematical physics. The lack of such a comprehensive theory means that each type of physical problem, and hence, each type of equation, requires a different analytical or numerical technique for its solution. Functional analysis has been applied to develop powerful techniques for analyzing and constructing generalized solutions to families of linear partial differential equations. However, the methods of functional analysis are limited in studying nonlinear partial differential equations, because such techniques are based on linear mathematical structures. Since linear mathematical structures do not describe the simplest nonlinearity (multiplication), it is not surprising that functional analysis has not yielded a comprehensive framework for studying generalized solutions of par- 
tial differential equations.

Many approaches have been used to attempt to define a useful multiplication operation on spaces of functionals applied in linear analysis to study differential equations. The motivation for multiplying functionals comes from a plethora of applications that occur in modeling physical problems: construction of generalized solutions of certain partial differential equations requires the multiplication of linear functionals. However, a fundamental theorem of Schwartz [1] shows that the standard algebraic product cannot be defined consistently in spaces of functionals, such as the distributions or generalized functions; all such methods will produce ambiguous results leading to logical and arithmetical contradictions. Because standard multiplication does not produce meaningful results, any product of generalized functions defined must be weaker than standard multiplication.

Over the last thirty years, E.E. Rosinger [2 - 6] and J.F. Colombeau [7, 8], working independently, have developed a theoretical framework for quotient algebras of generalized functions to analyze nonlinear partial differential equations. Their approach extends classical linear mathematics to nonlinear mathematics by defining multiplication on sets of generalized functions by embedding these linear spaces into abstract algebras of nonlinear generalized 
functions with weak operations of multiplication. The potential application of a theory of nonlinear generalized functions enabling the analysis and approximation of solutions to nonlinear partial differential equations is enormous; such a theory could impact almost all areas of science and technology.

Although it is probable that the existing theories of quotient algebras of generalized functions will yield important insights into the analysis and computation of certain classes of nonlinear field equations, significant limitations of these theories are known. Because any logically consistent operation of multiplication of generalized functions must be much weaker than the classical multiplication of numbers and functions, such a product of generalized functions will always yield large collections of abstract objects representing weak solutions of the problem under investigation. Basically problems with no classical weak solutions, such as the product, $\delta \cdot H$, are converted into problems with uncountably many generalized solutions. The fundamental question then becomes how to determine a physically observed weak solution from an infinitude of possible weak solutions.

To approximate solutions, Colombeau (see References $[7,8]$ ) constructed an explicit theory of nonlinear generalized functions. This theory has been applied to analyze and compute solutions to nonlinear partial differential 
equations. The logic of solving a differential equation in a space of Colombeau generalized functions follows the standard approach of functional analysis: a method is established for generating sequences to approximate solutions within a collection of nonlinear generalized functions and then a compactnesstype argument is applied to show that the approximating sequences actually converge to limits within the space of approximating objects. A resulting limit then represents a solution to the equation. A key difficulty in applying a space of Colombeau generalized functions is determining a specific representation for the products of generalized functions occurring in the differential equation. As Colombeau [9] and his coworkers [10, 11] have shown, different physical problems require different distinct representations for a product of generalized functions such as $\delta \cdot H$. The representation of the product is found by adding more information about the problem. Roughly speaking, the solution of a concrete problem via nonlinear generalized functions requires: the equations modeling the physics, the initial-boundary values, and the additional problem-specific physical information required to fix the product of generalized functions. For example, in continuum shock wave problems, the additional information is obtained by requiring the product of generalized functions to satisfy the standard shock wave jump conditions. 
A number of authors, including, Oberguggenberger and Todorov [12], and Hoskins and Sousa Pinto [13], have shown that versions of the Colombeau algebras of nonlinear generalized functions may be constructed using nonstandard analysis. Nonstandard analysis is a relatively new area of mathematics, which emerged from basic research in mathematical logic. The subject was discovered in the early 1960s by A. Robinson [14]. The main contribution of nonstandard analysis to mathematics is the extension of the real numbers $\mathbf{R}$ to the hyperreal numbers ${ }^{*} \mathbf{R}$ that contain infinitely small (infinitesimals) and infinitely large numbers. The infinitesimals in a hyperreal number system have the algebraic properties of standard numbers, which justifies the formal manipulations of infinitesimals that engineers and physicists often use. Moreover, the existence of distinct, infinitely large numbers that can be manipulated and used like finite numbers to solve problems provides a powerful new tool for applications. Nonstandard methods may be used to construct algebras of nonlinear generalized functions, or nonstandard analysis may be used directly to study specific problems involving multiplication of generalized functions. The second approach is considered here.

In this article, jump conditions are derived using nonstandard analysis for one-dimensional converging shock waves in a compressible, inviscid, perfect 
gas. A class of nonstandard Heaviside functions is applied to model the microstructure of the shock wave jump. The term microstructure refers to the distinct functions, which represent the flow variables such as specific volume, pressure, and velocity across a shock wave. A shock wave is governed by the standard conservation equations written in primitive form. A shock jump is then modeled to occur over an infinitesimal interval with nonstandard jump functions. The nonstandard jump functions applied in the subsequent analysis are defined as follows: for a standard jump function $\phi(x)$ with jump $[\phi] \equiv \phi_{r}-\phi_{l}$ at $x=0$, define a nonstandard jump function by

$$
{ }^{*} \phi(x) \equiv \phi_{l}+[\phi]^{*} H(x)
$$

where ${ }^{*} H(x)$ is a piecewise differentiable, nonstandard Heaviside function. Following Colombeau [9], each flow parameter is assumed to have a distinct nonstandard jump function associated with it at the shock. This means that each flow variable may have a different nonstandard Heaviside function or microstructure across a shock wave.

It is shown that if the various Heaviside functions for the flow parameters have their jumps located on the same infinitesimal interval, then the 
governing equations expressed in nonconservative form yield the relations between these Heaviside functions unambiguously. Strictly speaking, the mathematics of the product of nonstandard generalized functions is unambiguous; therefore, the use of nonstandard Heaviside functions removes some of the mathematical difficulties associated with products such as $\delta \cdot H$.

The present work was motivated by the authors' interest (see Baty et al., $[15,16])$ in understanding how to add physical information to specify products of generalized functions contained in differential equations with model problems with well-defined regions of solution discontinuity. Nonstandard analysis is applied to build explicit examples of the infinitesimal microstructure for converging shock waves. If the existence of the nonstandard Heaviside functions is assumed, the present development greatly simplifies the analysis and manipulation of products of generalized functions.

\section{Nonstandard Generalized Functions}

Nonstandard analysis studies the extension of number systems and function spaces to quotient spaces, which contain idealized elements that are infinitely large and infinitely small. For example, the real numbers, R, may be ex- 
tended to a hyperreal number system ${ }^{*} \mathbf{R}$ that is defined as a quotient space resulting from applying an equivalence relation $\sim$ on the set of sequences of real numbers, $\mathbf{R}_{\mathbf{s}}$, indexed by the natural numbers $N$

$$
{ }^{*} \mathbf{R} \equiv \mathbf{R}_{\mathbf{s}} / \sim
$$

The equivalence relation $\sim$ is defined by selecting an ultrafilter on $N$. A resulting nonstandard set ${ }^{*} \mathbf{R}$ may be shown to be a linearly ordered field. The role of the equivalence relation in Equation (2) is to add the idealized elements.

For the present analysis, let $S$ denote the real numbers or a set of functions such as the space of locally integrable functions, $\mathbf{L}_{\text {loc }}(\mathbf{R})$. Let $S^{\mathbf{R}+}$ be the set of nets $\left(f_{\epsilon}\right)$ in $S$ with parameter $\epsilon \in \mathbf{R}+$. The nonstandard extension of $S$ will then be defined to be the quotient space ${ }^{*} S \equiv S^{\mathbf{R}+} / \sim$ for a fixed equivalence relation. The use of nets provides a generalization of the definition of Equation (2). Further details of nonstandard extensions are available in many textbooks, articles and proceedings; for example see Hurd and Loeb [17] and Arkeryd, et al. [18]. 


\subsection{Predistributions of Generalized Functions}

In the early development of nonstandard analysis, hyperreal techniques were applied to generalized functions, Robinson [14], with application to quantum theory, Kelemen and Robinson [19]. Robinson noticed that any regularizing sequence of smooth functions used to represent the Dirac delta measure could be replaced by a single function with hyperreal parameters. A nonstandard Dirac delta measure results for any standard function $g$ that satisfies

$$
\int_{-\infty}^{\infty} g(x) \mathrm{d} x=1
$$

by defining the internal function,

$$
{ }^{*} \delta(x)=\Omega g(\Omega x)
$$

where $\Omega$ is any infinite hyperreal number. To see how the internal function

(4) acts like the delta measure, perform the integration

$$
\int_{-\infty}^{\infty} \Omega g(\Omega x)^{*} \phi(x) \mathrm{d} x \approx *^{*} \phi(0) \int_{-\epsilon}^{\epsilon} \Omega g(\Omega x) \mathrm{d} x \approx \phi(0)
$$


where $\epsilon=\epsilon(\Omega)$ is an infinitesimal constant depending on $\Omega, \phi \in D(\mathbf{R})$ is an arbitrary standard test function, and the equivalence relation $\approx$ means infinitesimally close. The result follows by taking the standard part of Equation (5).

The power of the linear theory of generalized functions comes from the fact that many distinct regularizing sequences of functions may be applied to produce unique functionals, such as the delta measure. It is well known that the standard theory of generalized functions may be extended to a nonstandard theory of generalized functions, Laugwitz [20], Richter [21] and Todorov and Vernaeve [22]. In standard constructions, the delta measure results from abstract limits of regularizing sequences; on the other hand, in nonstandard constructions, the functional whose standard part produces the same result as the delta measure can be represented by uncountably many internal hyperreal functions with distinct microstructures. These internal functions are called predistributions and are defined here for the space of locally integrable functions ${ }^{*} \mathbf{L}_{\mathbf{l o c}}(\mathbf{R})$. A function $f \in{ }^{*} \mathbf{L}_{\mathbf{l o c}}(\mathbf{R})$ is a called a predistribution if for each $n \in N_{0}$ it satisfies the following: i) $f^{(n)}$ is piecewise differentiable, ii) $f^{(n)} \in{ }^{*} \mathbf{L}_{\mathbf{l o c}}(\mathbf{R})$, and iii) there exists a generalized function $T \in D^{\prime}(\mathbf{R})$ such 
that

$$
\int_{-\infty}^{\infty} f^{(n)}(x)^{*} \phi(x) \mathrm{d} x \approx(-1)^{n}\left\langle T, \phi^{(n)}\right\rangle,
$$

for all test functions $\phi \in D(\mathbf{R})$ and all $n \in N_{0}$.

The analysis of shock wave jump conditions also requires the use of nonstandard Heaviside functions. Like the Dirac delta measure, the standard Heaviside function can be represented by uncountably many functions with distinct microstructures (predistributions of the Heaviside function). To introduce a predistribution of the Heaviside function, let $\epsilon_{1}$ and $\epsilon_{2}$ be two infinitesimal numbers, at least one of which is not zero, $\epsilon_{1} \approx \epsilon_{2}$ and $\epsilon_{1}<\epsilon_{2}$. An internal Heaviside function ${ }^{*} H(x)$ is defined as follows:

$$
{ }^{*} H(x)=\left\{\begin{array}{ll}
0 & \text { if } x \leq \epsilon_{1} \\
* h(x) & \text { if } \epsilon_{1}<x<\epsilon_{2} \\
1 & \text { if } x \geq \epsilon_{2}
\end{array} .\right.
$$

In Equation $(7)^{*} h(x)$ is assumed to be a piecewise differentiable function contained in ${ }^{*} \mathbf{L}_{\text {loc }}(\mathbf{R})$, not necessarily monotonically increasing, which satisfies

$$
{ }^{*} h\left(\epsilon_{1}\right) \approx 0 \text { and }{ }^{*} h\left(\epsilon_{2}\right) \approx 1 .
$$


As an example, the following function can be used for *h(x) when $x$ is in the open infinitesimal interval $(0, \epsilon)$ :

$$
{ }^{*} h(x)=1-\exp (-\Omega x)
$$

where $\Omega$ is any infinite hyperreal and $\epsilon=\epsilon(\Omega)$ is any infinitesimal hyperreal such that $\Omega \epsilon$ is an infinite hyperreal. Combining Equations (7) and (9) yields the nonstandard Heaviside function

$$
{ }^{*} H(x)= \begin{cases}0 & \text { if } x \leq 0 \\ 1-\exp (-\Omega x) & \text { if } 0<x<\epsilon \\ 1 & \text { if } x \geq \epsilon\end{cases}
$$

Notice that ${ }^{*} H(x)$ is a differentiable function on $(0, \epsilon)$ and that the derivative is the internal function

$$
{ }^{*} H^{\prime}(x)= \begin{cases}0 & \text { if } x \leq 0 \\ \Omega \exp (-\Omega x) & \text { if } 0<x<\epsilon, \\ 0 & \text { if } x \geq \epsilon\end{cases}
$$


which is a predistribution for the Dirac delta. For all standard test functions $\phi \in D(\mathbf{R})$ it may be shown that

$$
\int_{-\infty}^{\infty}{ }^{*} H^{\prime}(x)^{*} \phi(x) \mathrm{d} x \approx \phi(0)
$$

following Equation (5). For more detail about nonstandard Heaviside functions and their properties see Baty et al. [16].

\subsection{Products of Generalized Functions}

The Schwartz theorem showing that generalized functions cannot be multiplied is a manifestation of a basic structural limitation of algebraic mathematics. Following Rosinger [5], consider a mathematical structure $(\mathcal{F}, \cdot, \mathcal{D})$ consisting of a collection $\mathcal{F}$ of functions defined on $\mathbf{R}$ that may exhibit a finite number of localized discontinuities. The standard Heaviside function

$$
H(x)= \begin{cases}0 & \text { if } x<0 \\ 1 & \text { if } x>0\end{cases}
$$

is contained in $\mathcal{F}$. Let . and $D$ denote the standard multiplication and differentiation operators acting on the set $\mathcal{F}$. 
From Equation (13) it follows that

$$
H^{m}(x)=(H \cdot H \cdot H \cdot \ldots \cdot H)(x)=H(x)
$$

for all $m>1$. Then applying the derivative operator in the sense of generalized functions to Equation (14) yields

$$
m H^{m-1}(x) D H(x)=D H(x)
$$

which implies that

$$
m H^{m-1}(x)=1 \text { and so } H^{m-1}(x)=\frac{1}{m}
$$

for all $m>1$. But Equation (16) contradicts the definition of the standard Heaviside function (13). This contradiction occurs because the Heaviside function has a jump at $x=0$. Rosinger has shown that the mathematical structure $(\mathcal{F}, \cdot, \mathcal{D})$ is over-specified and discontinuity, multiplication and differentiation cannot be combined in the steps of a single derivation. The 
over-specification of $(\mathcal{F}, \cdot, \mathcal{D})$ may be repaired by requiring that

$$
H^{2}(x)=(H \cdot H)(x) \neq H(x) .
$$

From the definition of the nonstandard Heaviside function, Equation (7), it follows that

$$
{ }^{*} H^{2}(x)=\left\{\begin{array}{ll}
0 & \text { if } x \leq \epsilon_{1} \\
{ }^{*} h^{2}(x) & \text { if } \epsilon_{1}<x<\epsilon_{2} \\
1 & \text { if } x \geq \epsilon_{2}
\end{array},\right.
$$

which implies that

$$
{ }^{*} H^{2}(x) \neq{ }^{*} H(x)
$$

because ${ }^{*} h^{2}(x) \neq{ }^{*} h(x)$ for $\epsilon_{1}<x<\epsilon_{2}$. That is, giving the Heaviside function ${ }^{*} H(x)$ an infinitesimal microstructure removes the over-specification which leads to the contradiction of Equation (16). Equation (19) generalizes for all differentiable nonstandard Heaviside functions to the result that

$$
{ }^{*} H^{m}(x) \neq{ }^{*} H(x)
$$

for all $m>1$, removing the contradictions obtained by Equation (14). Also 
notice, that differentiating Equation (18) yields

$$
{ }^{*} H(x)^{*} H^{\prime}(x)=\left\{\begin{array}{ll}
0 & \text { if } x \leq \epsilon_{1} \\
{ }^{*} h(x)^{*} h^{\prime}(x) & \text { if } \epsilon_{1}<x<\epsilon_{2} \\
0 & \text { if } x \geq \epsilon_{2}
\end{array} .\right.
$$

As an example of a product of generalized functions, consider $\delta \cdot H$. The multiplication of the delta measure with the Heaviside function occurs in the analysis and computation of shock waves if the governing equations of motion are written in nonconservative form; $\delta \cdot H$ comes from the nonlinear terms, such as the convective acceleration term $u u_{x}$, for a discontinuity in the field variable, $u$. In the standard theory of generalized functions, the product $\delta \cdot H$ does not yield a well-defined functional, because the results depend on the representations of $\delta$ and $H$. To see this behavior, choose the predistributions of Equation (10) and

$$
{ }^{*} \delta(x)=\frac{\Omega}{\pi} \cdot \operatorname{sech}(\Omega x)
$$

for the Heaviside function and Dirac delta respectively. Now, consider the 
functional acting on any standard test function $\phi \in D(\mathbf{R})$

$$
\int_{-\infty}^{\infty}{ }^{*} \delta(x)^{*} H(x)^{*} \phi(x) \mathrm{d} x=\int_{0}^{\infty} \frac{\Omega}{\pi} \operatorname{sech}(\Omega x)(1-\exp (-\Omega x))^{*} \phi(x) \mathrm{d} x .
$$

Integrating Equation (23) then gives

$$
\int_{0}^{\infty} \frac{\Omega}{\pi} \operatorname{sech}(\Omega x)(1-\exp (-\Omega x))^{*} \phi(x) \mathrm{d} x \approx \phi(0)\left\{\frac{1}{2}-\frac{\ln 2}{\pi}\right\} .
$$

If other predistributions are selected for $\delta$ and $H$, the numerical value multiplying $\phi(0)$ in Equation (24) will, in general, be different.

In the numerical approximation of shock problems, the use of nonconservative forms of the equations of motion produce shock wave speeds that depend on the numerical scheme and not the physics of shock propagation. Nonconservative numerical simulations of one-dimensional shock problems are analogous to the result of Equation (24): the specific numerical values are fixed by the choice of the approximations for $\delta$ and $H$.

Next, consider the product $\delta \cdot H$, but now assume $\delta=H^{\prime}$. Then for any predistribution representing the delta measure, $H^{\prime}$ defined on the interval $(-\epsilon, \epsilon)$, choose hyperreal numbers $\Omega, \epsilon=\epsilon(\Omega)$ so that $\Omega$ is infinite, $\epsilon$ is infinitesimal and $\Omega \epsilon$ is an infinite hyperreal. Then for any standard test 
function $\phi \in D(\mathbf{R})$, the nonstandard functional equation for $\delta \cdot H$ implies

$$
\int_{-\infty}^{\infty}{ }^{*} \delta(x)^{*} H(x)^{*} \phi(x) \mathrm{d} x=\int_{-\infty}^{\infty}{ }^{*} H^{\prime}(x)^{*} H(x)^{*} \phi(x) \mathrm{d} x
$$

and

$$
\int_{-\infty}^{\infty}{ }^{*} \delta(x)^{*} H(x)^{*} \phi(x) \mathrm{d} x \approx \frac{1}{2} \phi(0)
$$

independent of the order of the operations used to evaluate the integral.

Equation (26) is consistent with the results obtained by the standard theory of generalized functions. This special case of the product $\delta \cdot H$ may be computed using only the function $H$ and multiplication and is not overspecified, since $\delta \cdot H$ is not differentiated. The differentiation of the Heaviside function is avoided by applying integration by parts. To this end, consider the standard version of Equation (25)

$$
\int_{-\infty}^{\infty} \delta(x) H(x) \phi(x) \mathrm{d} x=\int_{-\infty}^{\infty} \frac{d}{d x}\left(\frac{1}{2} H(x)\right) \phi(x) \mathrm{d} x
$$

which assumes that $H(x)=H^{2}(x)$. Equation (27) then yields

$$
\int_{-\infty}^{\infty} \frac{d}{d x}\left(\frac{1}{2} H(x)\right) \phi(x) \mathrm{d} x=-\frac{1}{2} \int_{0}^{\infty} \frac{d}{d x} \phi(x) \mathrm{d} x=\frac{1}{2} \phi(0),
$$


producing the same result as the standard part of Equation (26). This example is analogous to integrating the governing equations of motion modeling shock wave propagation in conservative form: the predicted shock wave speed will be independent of the numerical approximation. Notice that the standard calculation performed in Equations (27) and (28) depends on the order of the algebraic and analytical steps. Changing the order of the operations can produce an over-specified problem with ambiguous results. On the other hand, the nonstandard result of Equation (26) is independent of the order of operations.

\section{Shock Wave Jump Conditions}

\subsection{Equations of Motion}

The equations governing the motion of a one-dimensional, compressible, inviscid gas are given by

$$
\begin{gathered}
(\ln \rho)_{t}+u(\ln \rho)_{r}+u_{r}+\frac{\kappa u}{r}=0, \\
u_{t}+u u_{r}+\nu p_{r}=0,
\end{gathered}
$$




$$
e_{t}+u e_{r}+\nu p\left(u_{r}+\frac{\kappa u}{r}\right)=0
$$

where $\nu$ is the specific volume, $\rho$ is the density $\left(\equiv \frac{1}{\nu}\right), u$ is the velocity, $p$ is the pressure, and $e$ is the specific energy. The notation for partial differentiation is simplified to the subscript notation $\xi_{r} \equiv \frac{\partial \xi}{\partial r}$. Here $\kappa=0,1,2$ represents Cartesian, cylindrical and spherical coordinate systems, respectively. The conservation of mass, momentum and energy Equations (29), (30), and (31), represent three equations in terms of four physical variables. To close the system of equations two equations of state must be added. The equations of state relate the thermodynamic variables $\rho, p, e$, and $T$. Assuming a calorically perfect gas, the energy equation may be shown to be

$$
\frac{1}{\gamma-1}\left[(p \nu)_{t}+u(p \nu)_{r}\right]+\nu p\left(u_{r}+\frac{\kappa u}{r}\right)=0
$$

where $\gamma$ is the ratio of specific heats. Developments of Equations (29), (30), and (32) and discussions of the equations of state may be found in Zel'dovich and Raizer [23] and Thompson [24]. 


\subsection{Jump Conditions for Normal Shock Waves}

To motivate the physical assumptions used to derive jump conditions for converging shock waves, consider the motion of a normal shock wave $(\kappa=0)$ propagating through a perfect gas. On either side of the shock wave the specific volume $\nu$, velocity $u$, and pressure $p$ of the flow are assumed to be constant. Since the values of the field variables are assumed constant on either side of the shock wave, the shock wave does not accelerate and the shock speed $c$ is constant. Therefore, the characteristics for the shock wave are straight lines in the space-time domain. Along the characteristic lines the governing equations reduce to ordinary differential equations, so the field variables, $\nu, u$, and $p$, may be assumed to have the following form across the shock wave

$$
\begin{aligned}
& \nu(\xi)=\nu_{l}+[\nu] H(\xi), \\
& u(\xi)=u_{l}+[u] K(\xi), \\
& p(\xi)=p_{l}+[p] L(\xi),
\end{aligned}
$$

where $\xi=x+c t$ is a characteristic line, $c$ is the shock speed, and where $[\phi]=\phi_{r}-\phi_{l}$, with the subscripts $r$ and $l$ referring to the right (downstream) 
and left (upstream) conditions across the shock, respectively. The functions $H, K$, and $L$ are assumed to be predistributions of the Heaviside function as defined in Section 2.1. (Here and in the following development the $*_{-}$ notation is suppressed.) For an arbitrary fixed infinitesimal $\epsilon$, each one of these Heaviside functions is taken to have its jump contained on the same arbitrary infinitesimal interval $(0, \epsilon)$.

Now, substituting the jump functions (33), (34), and (35) into the equations of motion (29), (30), and (32) yields

$$
\begin{gathered}
\tilde{u}[\nu] H^{\prime}-\nu[u] K^{\prime}=0, \\
\tilde{u}[u] K^{\prime}+\nu[p] L^{\prime}=0, \\
\alpha \tilde{u}\left(p[\nu] H^{\prime}+\nu[p] L^{\prime}\right)+\nu p[u] K^{\prime}=0,
\end{gathered}
$$

where

$$
\tilde{u}=\left(u_{l}+c\right)+[u] K \text { and } \alpha=\frac{1}{\gamma-1} .
$$

Recasting Equations (36), (37), and (38) as a first-order system of ODEs 
generates the formal equation

$$
\left[\begin{array}{ccc}
{[\nu] \tilde{u}} & -[u] \nu & 0 \\
0 & {[u] \tilde{u}} & {[p] \nu} \\
\alpha[\nu] p \tilde{u} & {[u] p \nu} & \alpha[p] \tilde{u} \nu
\end{array}\right]\left[\begin{array}{c}
H \\
K \\
L
\end{array}\right]^{\prime}=\left[\begin{array}{l}
0 \\
0 \\
0
\end{array}\right],
$$

for the jump functions defining $\nu, u$, and $p$.

A nontrivial family of ODEs may be found by specifying the nonstandard Heaviside functions $H^{\prime}, K^{\prime}$, and $L^{\prime}$ if the determinant of the matrix of Equation (40) vanishes. The determinant of the matrix of Equation (40) is zero

$$
\left|\begin{array}{ccc}
{[\nu] \tilde{u}} & -[u] \nu & 0 \\
0 & {[u] \tilde{u}} & {[p] \nu} \\
\alpha[\nu] p \tilde{u} & {[u] p \nu} & \alpha[p] \tilde{u} \nu
\end{array}\right|=0
$$

if

$$
\tilde{u}^{2}=\gamma p \nu
$$

Equations (41) and (42) imply that a nontrivial algebraic solution exists defining the ODEs specifying the nonstandard Heaviside functions for isentropic flow. Since shock propagation is not an isentropic process, Equation (40) will not be satisfied across a shock wave; therefore, Equation (40) is 
replaced by the under-determined system

$$
\left[\begin{array}{ccc}
{[\nu] \tilde{u}} & -[u] \nu & 0 \\
0 & {[u] \tilde{u}} & {[p] \nu}
\end{array}\right]\left[\begin{array}{l}
H \\
K \\
L
\end{array}\right]^{\prime}=\left[\begin{array}{l}
0 \\
0
\end{array}\right]
$$

together with the entropy defined by

$$
s=c_{\nu} \ln \left(p \nu^{\gamma}\right)
$$

on either side of the shock wave, Zel'dovich and Raizer [23], p 53.

The jump functions for $\nu, u$, and $p$ are then determined by integrating matrix Equation (43) to determine the nonstandard Heaviside functions $H$, $K$, and $L$ on an infinitesimal interval, $(0, \epsilon)$, subject to boundary data

$$
\left[\begin{array}{c}
H(0) \\
K(0) \\
L(0)
\end{array}\right] \approx\left[\begin{array}{l}
0 \\
0 \\
0
\end{array}\right] \text { and }\left[\begin{array}{c}
H(\epsilon) \\
K(\epsilon) \\
L(\epsilon)
\end{array}\right] \approx\left[\begin{array}{c}
1 \\
1 \\
1
\end{array}\right]
$$

The boundary value problem defined by Equations (43) and (45) may be integrated to produce unambiguous relationships between the Heaviside 
functions $H, K$, and $L$. To integrate $H^{\prime}$ and $K^{\prime}$, Equation (43) is rewritten in terms of a nonsingular matrix and an unknown function depending on $L^{\prime}$

$$
\left[\begin{array}{cc}
{[\nu] \tilde{u}} & -[u] \nu \\
0 & {[u] \tilde{u}}
\end{array}\right]\left[\begin{array}{c}
H \\
K
\end{array}\right]^{\prime}=\left[\begin{array}{l}
f\left(L^{\prime}\right) \\
g\left(L^{\prime}\right)
\end{array}\right]
$$

where

$$
\left[\begin{array}{l}
f\left(L^{\prime}\right) \\
g\left(L^{\prime}\right)
\end{array}\right]=\left[\begin{array}{c}
0 \\
-[p] \nu L^{\prime}
\end{array}\right]
$$

Equations (46) and (47) then imply

$$
\left[\begin{array}{c}
H \\
K
\end{array}\right]^{\prime}=\frac{1}{[\nu][u] \tilde{u}^{2}}\left[\begin{array}{cc}
{[u] \tilde{u}} & {[u] \nu} \\
0 & {[\nu] \tilde{u}}
\end{array}\right]\left[\begin{array}{c}
0 \\
-[p] \nu L^{\prime}
\end{array}\right]
$$

where

$$
H^{\prime}=-\frac{[p] \nu^{2}}{[\nu] \tilde{u}^{2}} L^{\prime}
$$

and

$$
K^{\prime}=-\frac{[p] \nu}{[u] \tilde{u}} L^{\prime}
$$


Then combining Equations (49) and (50) yields

$$
H^{\prime}=\frac{[u] \nu}{[\nu] \tilde{u}} K^{\prime}
$$

Integrating Equation (51) and applying the boundary conditions (45) shows that

$$
H \approx K
$$

with

$$
\frac{\nu_{l}[u]}{[\nu] \tilde{u}_{l}}=1
$$

where

$$
\tilde{u}_{l}=u_{l}+c .
$$

Combining Equations (52) and (53) with Equation (50) produces

$$
K^{\prime}=-\frac{[p][\nu]}{[u]^{2}} L^{\prime}
$$

And integrating Equation (55) with the boundary conditions (45) yields

$$
K \approx L
$$


and

$$
\frac{[u]^{2}}{[\nu][p]}=-1
$$

Finally, by combining Equations (52) and (56) it follows that the microstructure for the Heaviside functions for the specific volume, velocity, and pressure jump conditions are coincident across an inviscid shock wave

$$
H \approx K \approx L,
$$

for an arbitrary infinitesimal interval $(0, \epsilon)$. This result also holds if the equivalence relation $\approx$ is replaced by $=$ in Equations (45) and (58). The result of Equation $(58)$ (with $=$ ) has been derived by Colombeau and Le Roux [10] using the theory of nonlinear generalized functions.

Equation (58) was derived by manipulating products of the functions, $H$, $K$, and $L$ and their derivatives. Because these functions satisfy (19), the mathematical structure applied in the analysis is not over-specified and can be used to derive the classical Rankine-Hugoniot jump conditions obtained by analyzing the problem in divergent form. For example, an expression for 
the shock speed $c$ follows from Equations (53) and (54), which yields

$$
c=\frac{1}{[\nu]}\left(\nu_{l}[u]-u_{l}[\nu]\right)
$$

Equation (59) is the shock speed obtained from the equations of motion in conservative form (which is the physically observed shock speed). A discussion of the standard one-dimensional shock jump conditions is given in Chapter 1 of Gathers [25].

In case the relaxation phenomenon is important in the shock wave propagation process, the infinitesimal length scale over which the jump in the nonstandard Heaviside functions takes place should correspond to the physical length over which of the slowest energy state reaches equilibrium. The physics of the relaxation phenomenon must be included in the differential equations to determine the microstructure of the Heaviside functions.

\subsection{Jump Conditions for Converging Shock Waves}

In analogy with the normal shock wave analysis of Section 3.2, the governing equations of motion are considered along characteristic curves in the spacetime domain. Along the characteristic curves the equations of motion reduce 
to ordinary differential equations. Following Zel'dovich and Raizer [23], the flow variables are assumed of the form

$$
p(\xi)=\rho_{0} \dot{R}^{2} \pi(\xi), \rho(\xi)=\rho_{0} g(\xi), \text { and } u(\xi)=\dot{R} v(\xi),
$$

where the characteristic curves are defined by

$$
\xi=\frac{r}{R}=\frac{r}{A(-t)^{\alpha}}
$$

In Equations (60) and (61), $R(\equiv R(t))$, the location of the shock wave, and $\rho_{0}$, the density in front of the shock wave, are the reference scales for the similarity solution; while $A$ and $\alpha$ are constants. The functions of Equations (60) and (61) are defined on

$$
-\infty<t \leq 0, R \leq r<\infty \text {, and } 1 \leq \xi<\infty .
$$

The converging shock fronts are located at $\xi=1$ and the shock speeds are given by $D \equiv \dot{R}=\alpha R / t$.

Recalling that shock wave propagation is not an isentropic process, Equations (29) and (30) are used to define ODEs relating the density $\rho$, velocity 
$u$, and pressure $p$ across converging shock waves. Because the resulting differential equations will be under-determined, unique jump functions cannot be found using these equations. However, the analysis will yield unambiguous relationships between the jump functions for the flow parameters. And, as in the case of the inviscid normal shock, if one of the jump functions is specified, the other two jump functions can be determined.

Along the characteristic curves, the similarity solutions $\pi, g$, and $v$ are then assumed to be internal nonstandard jump functions across a shock front

$$
\begin{aligned}
& g(\xi)=g_{0}+[g] G(\xi), \\
& v(\xi)=v_{0}+[v] K(\xi), \\
& \pi(\xi)=\pi_{0}+[\pi] L(\xi),
\end{aligned}
$$

In Equations (63), (64), and (65), each Heaviside predistribution is assumed to have its jump contained on the same interval $(1,1+\epsilon)$, where $\epsilon$ is an arbitrary fixed infinitesimal. Here $G, K$, and $L$ are assumed to be differentiable on $(1,1+\epsilon)$, and $G, K, L \in{ }^{*} \mathbf{L}_{\mathbf{l o c}}(\mathbf{R})$ as in Section 2.1. On the end points of the interval $(1,1+\epsilon)$ the nonstandard Heaviside functions are subject to the 
boundary data

$$
\left[\begin{array}{c}
G(1) \\
K(1) \\
L(1)
\end{array}\right] \approx\left[\begin{array}{l}
0 \\
0 \\
0
\end{array}\right] \text { and }\left[\begin{array}{c}
G(1+\epsilon) \\
K(1+\epsilon) \\
L(1+\epsilon)
\end{array}\right] \approx\left[\begin{array}{c}
1 \\
1 \\
1
\end{array}\right]
$$

Behind a converging shock front, the density $\rho_{1}$, velocity $u_{1}$, and pressure $p_{1}$, are assumed to take the limiting values for a strong shock

$$
\begin{aligned}
& \rho_{1}=\rho_{0} \frac{\gamma+1}{\gamma-1}, \\
& u_{1}=\frac{2}{\gamma+1} D,
\end{aligned}
$$

and

$$
p_{1}=\frac{2}{\gamma+1} \rho_{0} D^{2}
$$

Thompson [24], p 495; where $\gamma$ is the ratio of specific heats. Combining the general functional form of the similarity solutions (60), with the nonstandard jump functions (63) to (65), the Heaviside boundary data (66), and the physical boundary data (67) to (69), yields the nonstandard jump function 
parameters

$$
\begin{gathered}
g_{0}=1 \text { and }[g]=\frac{\gamma+1}{\gamma-1}-1, \\
v_{0}=0 \text { and }[v]=\frac{2}{\gamma+1},
\end{gathered}
$$

and

$$
\pi_{0}=0 \text { and }[\pi]=\frac{2}{\gamma+1}
$$

Now to determine relationships between the nonstandard Heaviside functions, $G, K$ and $L$, the equations of conservation of mass and momentum must be integrated. To this end, substituting Equations (60) into (29) and (30) produces the system of ODEs

$$
\left[\begin{array}{cc}
{[v]} & 0 \\
0 & {[\pi]}
\end{array}\right]\left[\begin{array}{c}
K \\
L
\end{array}\right]^{\prime}=\left[\begin{array}{l}
f(G) \\
g(G)
\end{array}\right]
$$

where

$$
\left[\begin{array}{c}
f(G) \\
g(G)
\end{array}\right]=\left[\begin{array}{c}
\xi(\ln g)^{\prime}-\left(\kappa / \xi+(\ln g)^{\prime}\right) v \\
-g\left([(\alpha-1) / \alpha] v+(v-\xi) v^{\prime}\right)
\end{array}\right]
$$

The system of Equations (73) have assumed that combinations of $R, \rho$ and their time derivatives are functions of the constant $\alpha$. Details of the derivation of these equations and the scale factor identities are given in Chapter 
XII of Zel'dovich and Razier [23].

The first Equation of (73) may be integrated to solve for either $g$ or $v$. Since this equation forms a linear ODE in terms $g$,

$$
\frac{d v}{d \xi}+\left(\frac{\kappa}{\xi}+(\ln g)^{\prime}\right) v=\xi(\ln g)^{\prime}
$$

where $(\cdot)^{\prime} \equiv \frac{d}{d \xi}, v$ is determined as a function $g$. Integrating Equation (74) on the interval $(1,1+\epsilon)$ yields

$$
v(\xi)=\frac{1}{\xi^{\kappa} g(\xi)}\left[\xi^{\kappa+1} g(\xi)-1-(\kappa+1) \int_{1}^{\xi} \tau^{\kappa} g(\tau) d \tau\right] .
$$

To show that Equation (76) gives a jump function, it must be demonstrated that it satisfies the boundary conditions of Equations (66) and (71). From experimental shock data, $g$ is assumed to be strictly monotonically increasing on the interval $(1,1+\epsilon)$; with this assumption the integral in Equation (76) satisfies

$$
0<(\kappa+1) \int_{1}^{\xi} \tau^{\kappa} g(\tau) d \tau \leq(\kappa+1) g(1+\epsilon) \int_{1}^{\xi} \tau^{\kappa} d \tau
$$


But recalling that

$$
(1+\epsilon)^{k} \approx 1 \text { for } k>0,
$$

implies that

$$
0<(\kappa+1) \int_{1}^{\xi} \tau^{\kappa} g(\tau) d \tau \leq \omega
$$

where $\omega$ is an infinitesimal. Equations (76) and (79) then yield

$$
v(\xi) \approx \frac{1}{\xi^{\kappa} g(\xi)}\left[\xi^{\kappa+1} g(\xi)-1\right] .
$$

and

$$
v(1+\epsilon) \approx \frac{2}{\gamma+1},
$$

so together with $v(1) \approx 0$, it follows from Equation (64) that $v$ is a nonstandard jump function. Equation (80) is the homogeneous part of the solution of Equation (75), which implies that the nonhomogeneous term generates an infinitesimal contribution to the velocity jump function across the shock wave.

Next, the second Equation in (73) gives a linear ODE for $\pi$ in terms of $v$ and $g$

$$
\frac{d \pi}{d \xi}=-g\left(\frac{\alpha-1}{\alpha} v+(v-\xi) v^{\prime}\right)
$$


Integrating Equation (82) on the interval $(1,1+\epsilon)$ produces

$$
\pi(\xi)=-\frac{\alpha-1}{\alpha} \int_{1}^{\xi} g(\tau) v(\tau) d \tau-\int_{1}^{\xi} g(\tau)(v(\tau)-\tau) v^{\prime}(\tau) d \tau
$$

By combining Equations (76) and (83), $\pi$ is determined as a function of $g$. To simplify the resulting expression for $\pi$ in terms of $g$, Equation (76) is replaced by Equation (80) in Equation (83), which yields

$$
\pi(\xi) \approx-\frac{\alpha-1}{\alpha} \int_{1}^{\xi}\left(\tau g(\tau)-\frac{1}{\tau^{\kappa}}\right) d \tau+\int_{1}^{\xi} \tau^{-\kappa} v^{\prime}(\tau) d \tau
$$

Using Equations (77) to (79), the first integral in Equation (84) is bounded by an infinitesimal, so that Equation (84) becomes

$$
\pi(\xi) \approx \int_{1}^{\xi} \tau^{-\kappa} v^{\prime}(\tau) d \tau
$$

Integrating Equation (85) by parts then yields

$$
\pi(\xi) \approx \xi^{-\kappa} v(\xi)-v(1)+\kappa \int_{1}^{\xi} \tau^{-\kappa-1} v(\tau) d \tau
$$

Note that the integral term in Equation (86) is bounded by an infinitesimal, 
evaluating Equation (86) at $\xi=1$ and $\xi=1+\epsilon$ gives

$$
\pi(1) \approx 0 \text { and } \pi(1+\epsilon) \approx \frac{2}{\gamma+1}
$$

so $\pi$ is a nonstandard jump function. And since Equation (78) implies that

$$
\frac{1}{\xi^{\kappa}} \approx 1
$$

on the interval $(1,1+\epsilon)$, Equation (86) then becomes

$$
\pi(\xi) \approx v(\xi)
$$

By combining Equations (63) to (65), (67) to (69), and Equation (89), it follows that the predistributions of the Heaviside functions $G, K$, and $L$ for the density, velocity, and pressure jump conditions are in general distinct, across an inviscid shock wave

$$
G \neq K \neq L
$$


and

$$
K \approx L
$$

for any infinitesimal interval $(1,1+\epsilon)$. Moreover, the jump functions $v, \pi$ and $g$ are related by the integral equations

$$
v(\xi)=\frac{1}{\xi^{\kappa} g(\xi)}\left[\xi^{\kappa+1} g(\xi)-1-(\kappa+1) \int_{1}^{\xi} \tau^{\kappa} g(\tau) d \tau\right]
$$

and

$$
\pi(\xi)=-\frac{\alpha-1}{\alpha} \int_{1}^{\xi} g(\tau) v(\tau) d \tau-\int_{1}^{\xi} g(\tau)(v(\tau)-\tau) v^{\prime}(\tau) d \tau
$$

The nonstandard functions $g, v, \pi \in{ }^{*} \mathbf{L}_{\mathbf{l o c}}(\mathbf{R})$ are solutions of the boundary value problem defined by Equations (73) and (74) and Equations (63) through $(66)$ in the sense that the infinitesimal equivalence relation $\approx$ replaces equality $=$ in the equations of motion.

\section{Examples}

To exhibit examples for the microstructures of inviscid, converging shock waves, assume a density jump function $g(\xi) \in{ }^{*} \mathbf{L}_{\mathbf{l o c}}(\mathbf{R})$ defined via Equations 
(63) and (70) is given by

$$
g(\xi)=1+[g]\left(\frac{\xi-1}{\epsilon}\right)^{n}
$$

on the interval $(1,1+\epsilon)$, for $n \geq 1$. Also, assume the ratio of specific heats is given by $\gamma=7 / 5$ for all examples.

Substituting Equation (94) into Equation (76) then yields

$$
v(\xi)=\xi-\frac{1}{\xi^{\kappa} g(\xi)}-\frac{\kappa+1}{\xi^{\kappa} g(\xi)} \int_{1}^{\xi}\left[\tau^{\kappa}+\tau^{\kappa}[g]\left(\frac{\tau-1}{\epsilon}\right)^{n}\right] d \tau .
$$

Fixing $n=1$ and performing the integration in Equation (95) produces

$$
\begin{aligned}
v(\xi)=\xi-\frac{1}{\xi^{\kappa} g(\xi)}-\frac{\kappa+1}{\xi^{\kappa} g(\xi)} & \left.\left(1-\frac{[g]}{\epsilon}\right) \frac{\xi^{\kappa+1}}{\kappa+1}+\frac{[g]}{\epsilon} \frac{\xi^{\kappa+2}}{\kappa+2}\right) \\
+ & \frac{\kappa+1}{\xi^{\kappa} g(\xi)}\left(\left(1-\frac{[g]}{\epsilon}\right) \frac{1}{\kappa+1}+\frac{[g]}{\epsilon} \frac{1}{\kappa+2}\right) .
\end{aligned}
$$

By using Equations (66), (71), and (78) with Equation (96), $v$ satisfies the boundary conditions and is a nonstandard jump function for velocity

$$
v(1) \approx 0 \text { and } v(1+\epsilon) \approx \frac{2}{\gamma+1}
$$




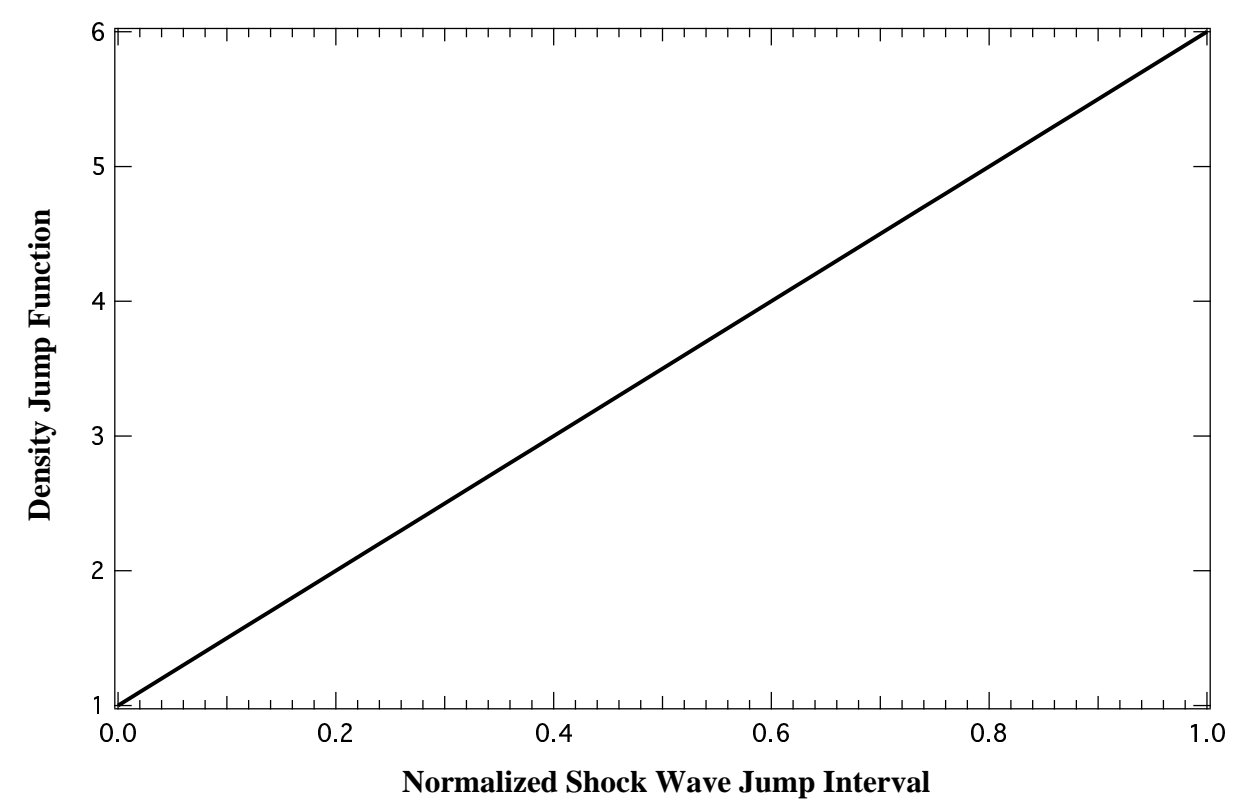

Figure 1: Density jump function specified form Equation (98) for $n=1$.

The jump function for pressure may be computed directly by combining Equations (94) and (96) with Equation (83) and integrating. However, the resulting form for the pressure contains many terms; so to simplify the analysis, the pressure may be integrated numerically for the case of a converging spherical shock wave, $\kappa=2$.

Figure 1 shows the density jump function specified by Equation (94) for $n=1$. The shock wave jump interval $(1,1+\epsilon)$ is normalized to the standard interval $(0,1)$ for all of the plots. The plots are generated with small real values for $\epsilon$ and are used to convey the qualitative behavior of the 


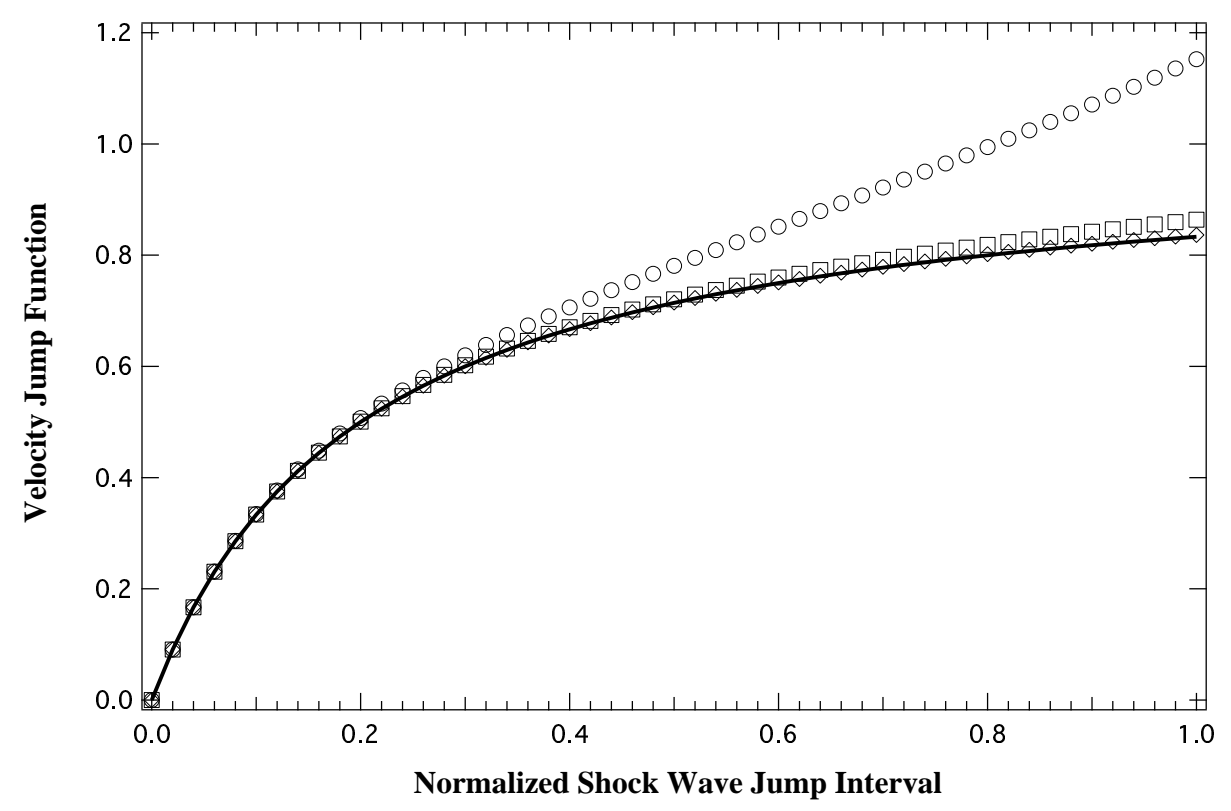

Figure 2: Computed velocity jump function for the density jump function shown in Figure 1: Circles $\epsilon=0.1$, Boxes $\epsilon=0.01$, Diamonds $\epsilon=0.001$, Solid line limiting solution.

nonstandard shock wave microstructure.

Figure 2 shows the velocity jump function $v$ computed from Equations (94) and (76). Approximations of the velocity are computed using Equation (80) for the values of $\epsilon=0.1,0.01,0.001$ and illustrate the asymptotic behavior the nonstandard jump functions. Moreover, as $\epsilon$ approaches an infinitesimal, the jump function for pressure approaches the jump function for velocity: $\pi \approx v$, and hence, $K \approx L$. As a second example, the microstructure is computed numerically for the case of a converging cylindrical shock 


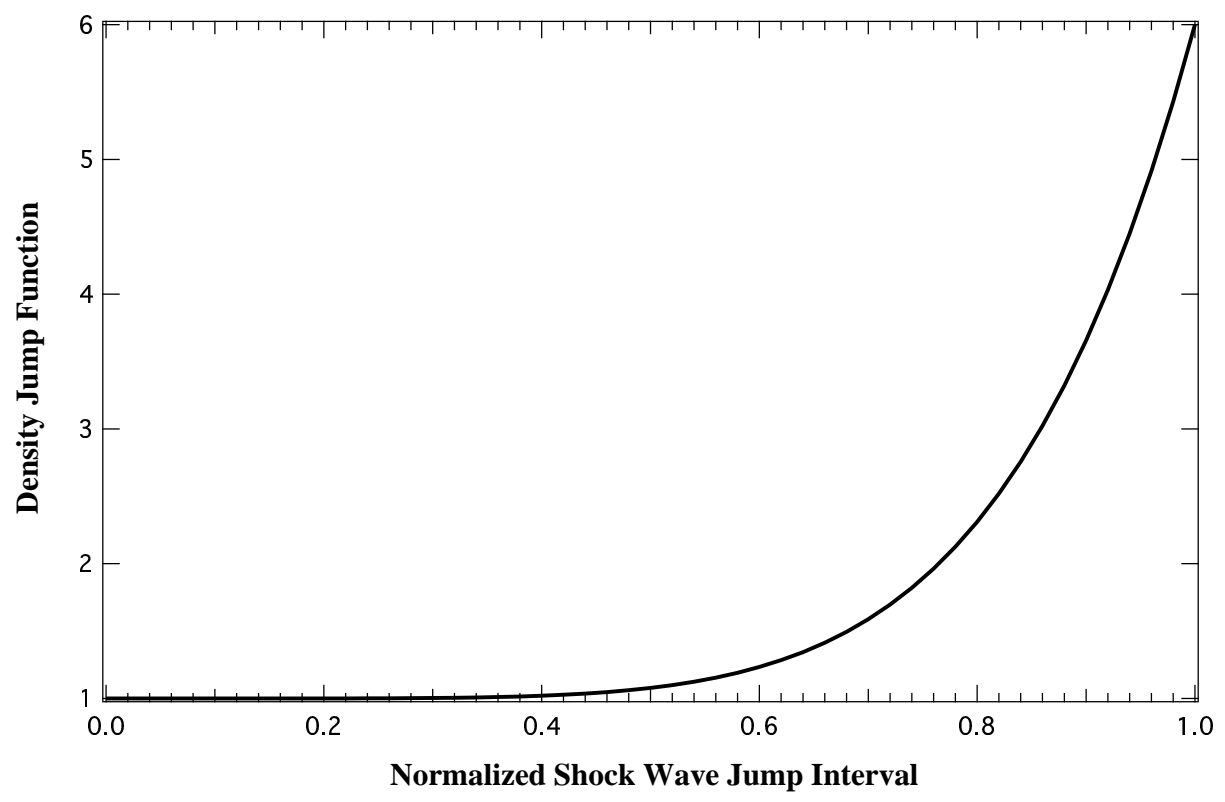

Figure 3: Density jump function specified form Equation (98) for $n=6$.

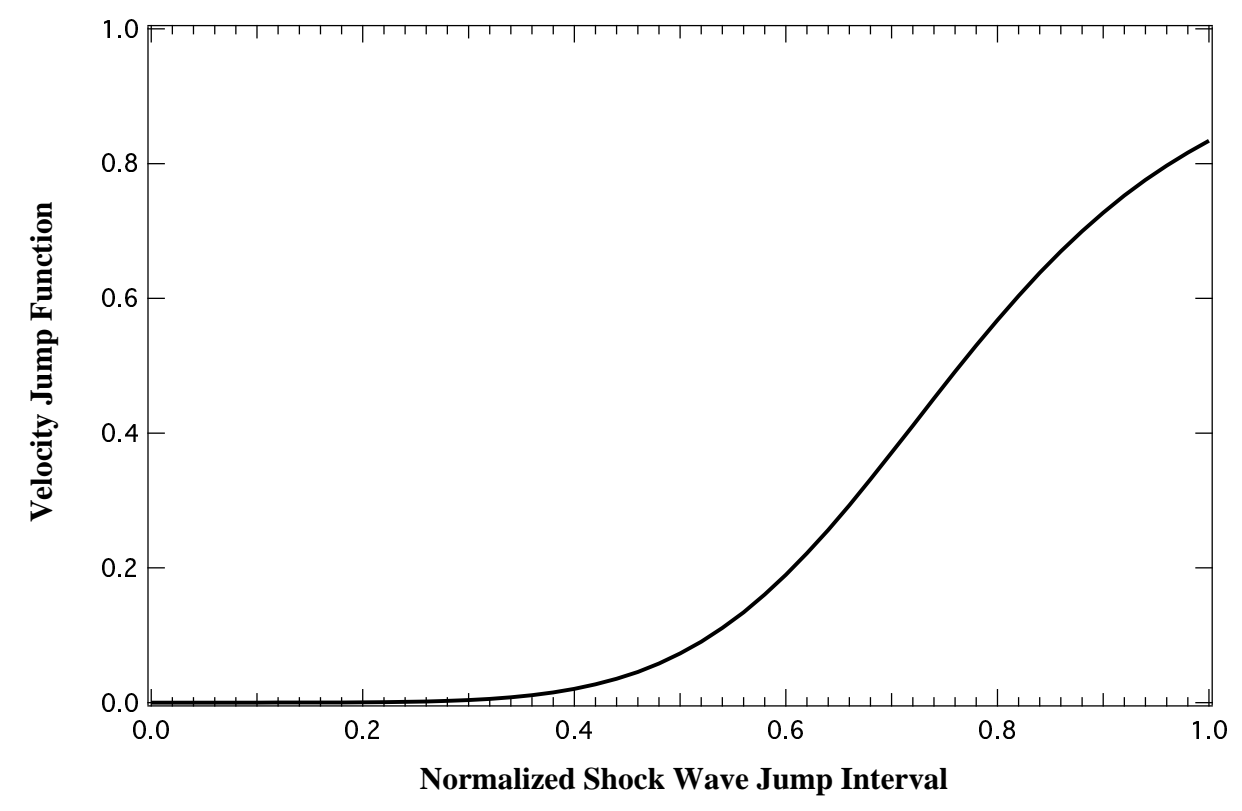

Figure 4: Computed velocity jump function for the density jump function shown in Figure 3: limiting solution $\epsilon \sim 0$. 
wave, $\kappa=1$. From experimental data on normal shock waves, the field variables have small variations near the upstream boundary of the shock layer, Muntz and Harnett [26]. To simulate this behavior, Equation (94) is considered with $n=6$, so that $g^{\prime}(1)$ is small. Figure 3 shows the density jump function $g$ on the normalized interval $(0,1)$. The velocity jump function is then computed using Equation (76), and exhibited in Figure 4. Because the asymptotic behavior of the nonstandard Heaviside function for pressure implies that $K \approx L$, it follows that Figure 4 also represents the pressure jump function $\pi$.

The microstructure of converging shock waves depends on the geometrical and physical parameters, $\alpha, \kappa$, and $\gamma$. The similarity parameter $\alpha$ appears in an integral term of Equation (83) for the pressure jump function $\pi$. Because this term is bounded by an infinitesimal, $\alpha$ has little effect on the basic features of the inviscid shock microstructure and is neglected in the examples. Nonzero variation of the geometrical term $\kappa$, which specifies the coordinate system (either 1 or 2), also has little effect on the gross microstructure. However, $\kappa$ was used to compute the examples and generates a small but noticeable variation for larger values of $\epsilon$. The ratio of specific heats $\gamma$ is the key physical parameter used to specify the macroscopic boundary conditions; 
and as such, $\gamma$ fixes the gross features of the computed microstructure of converging shock waves for a given density jump function.

\section{$5 \quad$ Summary and Conclusions}

This paper derived shock wave jump conditions for one-dimensional, converging shock waves in an inviscid, perfect gas using nonstandard analysis. Predistributions of the Heaviside functions were introduced and used to model the microstructure of the flow field across a shock wave governed by the classical conservation equations. For flow variables with their jumps defined on the same infinitesimal interval, it was shown that the equations of motion written in nonconservative form produced unambiguous relations between the various nonstandard Heaviside functions. Because inviscid flow was assumed, the analysis only produced the relations between the field variable Heaviside functions.

The present work was motivated by the authors' interest in understanding how to add physical information to specify products of generalized functions contained in differential equations modeling physical problems with well defined regions of solution discontinuity. The main conclusions of the study are 
as follows:

1. Nonstandard analysis may be applied to identify predistributions of the Heaviside function defined on infinitesimal intervals that reproduce the standard jump function results from the theory of generalized functions. The predistributions considered here are piecewise differentiable elements of the function space ${ }^{*} \mathbf{L}_{\text {loc }}(\mathbf{R})$.

2. Nonstandard asymptotic arguments were used to simplify the derivation of the shock wave jump functions.

3. The relationships between the internal Heaviside functions for one dimensional, converging shock waves in an inviscid, perfect gas were derived unambiguously from the equations of motion in nonconservative form. Distinct nonstandard jump functions were obtained for the density, velocity, and pressure in converging shock waves.

\section{Acknowledgments}

The authors would like to thank an anonymous reviewer for suggestions that clarified the mathematical framework used in the derivation of the nonstandard shock wave jump conditions. 


\section{References}

[1] Schwartz, L, Sur l'impossibility de la multiplication des distribution, Comptes Rendus de l'Academie des Sciences, Paris, 239, pp. 847-848, 1954.

[2] Rosinger, E. E., Distributions and Nonlinear Partial Differential Equations, Lecture Notes in Mathematics, 684, Springer-Verlag, 1978.

[3] Rosinger, E. E., Nonlinear Partial Differential Equations: Weak and Sequential Solutions, North-Holland Mathematics Studies, 44, Elsevier Science Publishers, 1980.

[4] Rosinger, E. E., Generalized Solutions of Nonlinear Partial Differential Equations, North-Holland Mathematics Studies, 146, Elsevier Science Publishers, 1987.

[5] Rosinger, E. E., Non-Linear Partial Differential Equations: An Algebraic View of Generalized Solutions, North-Holland Mathematics Studies, 164, Elsevier Science Publishers, 1990.

[6] Rosinger, E. E., Characterization for the Solvability of Nonlinear Partial Differential Equations, Transactions of the American Mathematical 
Society, 330, No. 1, pp. 203-225, 1992.

[7] Colombeau, J. F., New Generalized Functions and Multiplication of Distributions, North-Holland Mathematics Studies, 84, Elsevier Science Publishers, 1984.

[8] Colombeau, J. F., Elementary Introduction to New Generalized Functions, North-Holland Mathematics Studies, 113, Elsevier Science Publishers, 1985.

[9] Colombeau, J. F., The Elastoplastic Shock Problem as an Example of the Resolution of Ambiguities in the Multiplication of Distributions, Journal of Mathematical Physics, 30, No. 10, pp. 2273-2279, 1989.

[10] Colombeau, J. F., and Le Roux, A. Y., Multiplication of Distributions in Elasticity and Hydrodynamics, Journal of Mathematical Physics, 29, No. 2, pp. 315-319, 1988.

[11] Colombeau, J. F., Le Roux, A. Y., Noussair, A., Perrot, B., Microscopic Profiles of Shock Waves and Ambiguities in Multiplications of Distributions, SIAM Journal of Numerical Analysis, 26, No. 4, pp. 871-883, 1989. 
[12] Oberguggenberger, M., and Todorov, T. An Embedding of Schwartz Distributions in the Algebra of Asymptotic Functions, International Journal of Mathematics and Mathematical Sciences, 21, No. 3, pp. 417-428, 1998.

[13] Hoskins, R. F., and Sousa Pinto, J., Nonstandard Treatment of New Generalized Functions, in Generalized Functions and Their Applications, Edited by R. S. Pathak, Plenum Press, pp. 95-103, 1993.

[14] Robinson, A., Nonstandard Analysis, North-Holland, 1966.

[15] Baty, R. S., Vaughn, M. R., and Farassat, F., A Nonstandard Analysis of a Simple Discontinuous Force Equation Modelling Continuous Motion, Journal of Sound and Vibration, 202, No. 2, pp. 288-297, 1997.

[16] Baty, R. S., Farassat, F., and Hargreaves, J. A., Nonstandard Analysis and Shock Wave Jump Conditions in a One-Dimensional Compressible Gas, LA-14334, Los Alamos National Laboratory, May 2007, Electronic copy available: http://www.doe.gov/bridge.

[17] Hurd, A. E., and Loeb, P. A., An Introduction to Nonstandard Real Analysis, Academic Press, 1985. 
[18] Arkeryd, L. O., Cutland, N. J., and Henson, C. W., Nonstandard Analysis Theory and Application, NATO ASI Series, Series C: Mathematical and Physical Sciences, 493, Kluwer Academic Publishers, 1997.

[19] Keleman, P. J., and Robinson, A., The Nonstandard $\lambda: \phi_{2}^{4}(x)$ : Model I. The Technique of Nonstandard Analysis in Theoretical Physics, Journal of Mathematical Physics, 13, No. 12, pp. 1870-1874, 1972.

[20] Laugwitz, D., Infinitesimals in Physics (An Introduction to the Application of Nonstandard Analysis Methods), Mathematical Structures Computational Mathematics - Mathematical Modelling, 2, Papers Dedicated to Professor L. Iliev's 70th Anniversary, Sofia, pp. 233-243, 1984.

[21] Richter, M. M., Ideale Punkte, Monaden und Nichstandard-Methoden, Friedr. Vieweg and Sohn, 1982.

[22] Todorov, T. D., and Vernaeve, H. Full Algebra of Generalized Functions and Non-Standard Asymptotic Analysis, Unpublished Manuscript, arXiv:0712.2603v1, 2007.

[23] Zel'dovich, Ya. B., and Raizer, Yu. P. Physics of Shock Waves and HighTemperature Hydrodynamic Phenomena, Academic Press, 1966 (Volume I) 1967 (Volume II). 
[24] Thompson, P. A., Compressible-Fluid Dynamics, McGraw-Hill, 1972.

[25] Gathers, G. R., Selected Topics in Shock Wave Physics and Equation of State Modeling, World Scientific, 1994.

[26] Muntz, E. P., and Harnett, L. N., Molecular Velocity Distribution Function Measurements in a Normal Shock Wave, The Physics of Fluids, 12, No. 10, pp. 2027-2035, 1969. 